\title{
NEW HIGH-PRECISION VALUES OF THE GEODETIC ROTATION OF THE MAJOR PLANETS, PLUTO, THE MOON AND THE SUN
}

\author{
V.V. Pashkevich \\ Central (Pulkovo) Astronomical Observatory of RAS \\ Pulkovskoe shosse, 65/1, 196140, St.Petersburg, Russia \\ e-mail: pashvladvit@yandex.ru
}

\begin{abstract}
This investigation is continuation of our studies of the geodetic (relativistic) rotation of the Solar system bodies (Eroshkin and Pashkevich, 2007) and (Eroshkin and Pashkevich, 2009). For each body (the Moon, the Sun, the major planets and Pluto) the files of the values of the components of the angular velocity of the geodetic rotation are constructed over the time span from AD1000 to AD3000 with one day spacing, by using DE422/LE422 ephemeris (Folkner, 2011), with respect to the proper coordinate systems of the bodies (Seidelmann et al., 2005). For the first time in the perturbing terms of the physical librations for the Moon and in Euler angles for other bodies of the Solar system the most essential terms of the geodetic rotation are found by means of the least squares method and spectral analysis methods.
\end{abstract}

Keywords: geodetic (relativistic) rotation, angular velocity vector, Solar system bodies, reference frame, the major planets, Pluto, the Moon, the Sun, ephemeris.

\section{INTRODUCTION}

The geodetic rotation of the body is a most essential relativistic effect it's rotation. It is effect consist of two effects: the geodetic precession is the systematic effect and the geodetic nutation is the periodic effect. These effects have some analogies with precession and nutation, which are better-known events on the classical mechanics. Their emergence, unlike the last classical events, are not depend on from influences of any forces to body, represents only the effect of the curvature of space-time, predicted by general relativity, on a vector of the body rotation axis carried along with an orbiting body.

In the previous investigations (Eroshkin and Pashkevich, 2007) and (Eroshkin and Pashkevich, 2009) the problem of the geodetic (relativistic) rotation of the major planets, Pluto, the Moon and the Sun were studied by using the DE404/LE404 ephemeris (Standish et al., 1995). The most essential terms of the geodetic rotation in the projections of the angular velocity vector of the geodetic rotation for the body of Solar system were found. 
The main purposes of the present research:

1. To obtain a new method for calculation the values of the geodetic rotation of any bodies of the Solar system.

2. In the first time for the perturbing terms of the physical librations of the Moon and for Euler angles of the other Solar system bodies to define new high-precision values of the geodetic rotation of the dynamically adjusted to the DE422/LE422 ephemeris (Folkner, 2011).

\section{METHOD OF THE PROBLEM SOLUTION}

In order to attain these aims the problem is studied by means new method for calculation the values of the geodetic rotation of any bodies of the Solar system:

A) The problem of the geodetic (relativistic) rotation of the Solar system bodies (the Moon, major planets, Pluto and the Sun) is studied with respect to the proper coordinate systems of the bodies (Seidelmann et al., 2005).

B) The values of the velocities geodetic rotation are determined (in the perturbing terms of the physical librations for the Moon and in Euler angles for others Solar system bodies) over the time span from AD1000 to AD3000 with one day spacing by using the DE422/LE422 ephemeris.

C) The most essential terms of the geodetic rotation are found by means of the least squares method and spectral analysis methods.

A. The problem of the geodetic (relativistic) rotation of the Solar system bodies (the Moon, major planets, Pluto and the Sun) is studied with respect to the proper coordinate systems of the bodies (Seidelmann et al., 2005). The positions and velocities of the bodies are defined by means the DE422/LE422 ephemeris.

It is well known that the vector of the angular velocity of the geodetic rotation of a body $i$, which is the most essential relativistic component of the body rotational motion around the proper center of mass, is defined by the following expression (Eroshkin and Pashkevich, 2009):

$$
\bar{\sigma}_{i}=\frac{1}{c^{2}} \sum_{j \neq i} \frac{G m_{j}}{\left|\bar{R}_{i}-\bar{R}_{j}\right|^{3}}\left(\bar{R}_{i}-\bar{R}_{j}\right) \times\left(\frac{3}{2} \dot{\bar{R}}_{i}-2 \dot{\bar{R}}_{j}\right) .
$$

Here $c$ is the velocity of light; $G$ is the gravitational constant; $m_{j}$ is the mass of a body $j$; $\bar{R}_{i}, \dot{\bar{R}}_{i}, \bar{R}_{j}, \dot{\bar{R}}_{j}$ are the vectors of the barycentric position and velocity of bodies $i$ and $j$. The symbol $\times$ means a vector product; the subscripts $i$ and $j$ correspond to the Moon, the major planets, Pluto and the Sun. 


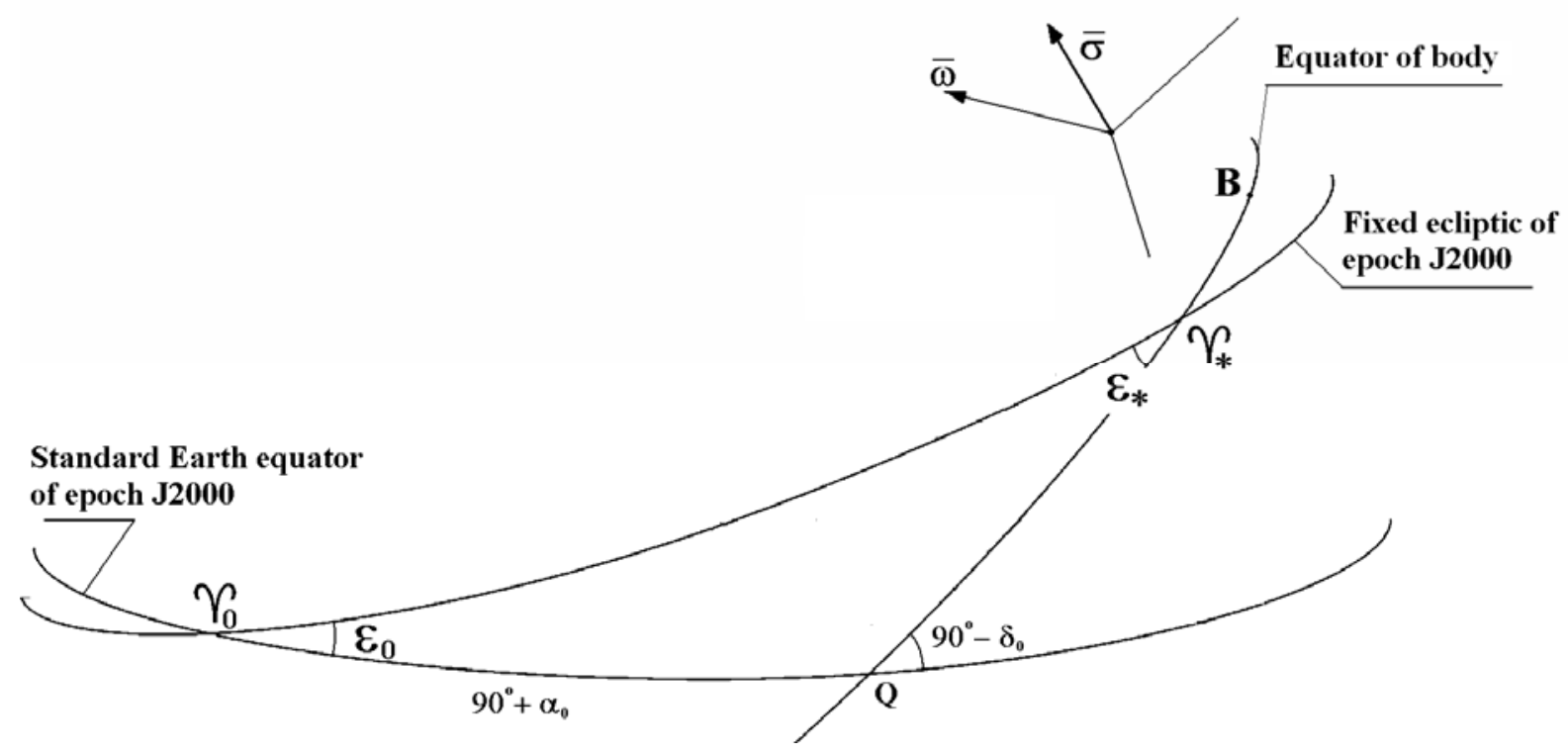

Fig. 1. Triangle used to define the direction of the angular velocity vector of the geodetic rotation for any body of Solar system

The relativistic angular velocity vector for any Solar system bodies is calculated as follows:

$$
\bar{\omega}_{R}=\bar{\omega}+\bar{\sigma} .
$$

Here and further the body index $i$ is omitted; $\bar{\omega}$ is Newtonian angular velocity vector for any Solar system bodies.

The reduction of the components of the angular velocity vector of the geodetic rotation of the Solar system bodies, from the geocentric reference frame (the reference frame of DE422/LE422 ephemeris (Folkner, 2011)) to the body-centric reference frames, given by Seidelmann et al. (2005), are found from the triangle (presented in Figure 1), which is used to define the direction of the angular velocity vector of the geodetic rotation for any body of the Solar system, by means of the following formulae:

$$
\begin{aligned}
& \cos \varepsilon_{*}=\sin \delta_{0} \cos \varepsilon_{0}-\cos \delta_{0} \sin \varepsilon_{0} \sin \alpha_{0}, \\
& \sin \gamma_{0} \gamma_{*}=\frac{\cos \delta_{0} \cos \alpha_{0}}{\sin \varepsilon_{*}}, \sin \mathrm{Q} \gamma_{*}=\frac{\sin \varepsilon_{0} \cos \alpha_{0}}{\sin \varepsilon_{*}}, \\
& \varphi=\mathrm{W}-\mathrm{Q} \gamma_{*}+180^{\circ}, \theta=-\varepsilon_{*}, \gamma_{*} \mathrm{~B}=\mathrm{W}-\mathrm{Q} \gamma_{*},
\end{aligned}
$$

and by means of the following matrix product:

$$
\left(\begin{array}{c}
\sigma_{1} \\
\sigma_{2} \\
\sigma_{3}
\end{array}\right)=r\left(\gamma_{*} \mathrm{~B}\right) p\left(-\varepsilon_{*}\right) r\left(\gamma_{0} \gamma_{*}\right) p\left(\varepsilon_{0}\right) r(\Delta)\left(\begin{array}{c}
\sigma_{X} \\
\sigma_{Y} \\
\sigma_{Z}
\end{array}\right),
$$

where $\sigma_{X}, \sigma_{Y}, \sigma_{Z}$ are the components of the geocentric vector of the angular velocity of the geodetic rotation of a body; $\sigma_{1}, \sigma_{2}, \sigma_{3}$ are the components of the body-centric vector of the angular velocity of the geodetic rotation of a body; $X, Y, Z$ are the components of the vector $\bar{R}$ in the expression (1); $\varepsilon_{0}=23^{\circ} 26^{\prime} 21^{\prime \prime} .40928$ and $\Delta=-0 " .05294$ are taken from Bretagnon et al. (1998) (the equatorial coordinate system of DE422/LE422 ephemeris is put to the fixed ecliptic and dynamical equinox J2000 in the result of two rotations: a rotation in the equator plane 
at the angle $\Delta$ to the point of the dynamical equinox $\mathrm{J} 2000$ and the rotation at the angle $\varepsilon_{0}$ to the plane of the fixed ecliptic J2000.0); $\alpha_{0}$ is the right ascension of the north pole of body rotation; $\delta_{0}$ is the declination of the north pole of body rotation; angle $\mathrm{W}=\mathrm{QB}$ specifies the location of the prime meridian of the body, which is measured along the equator of body an easterly direction with respect to the north pole of body from the node Q (located at right ascension $90^{\circ}+\alpha_{0}$ ) of the equator of body on the standard Earth equator of epoch J2000 to the point $B$, where the prime meridian crosses the equator of body (see Figure 1) (recommended values of the constants in the expressions for $\alpha_{0}, \delta_{0}$ and $\mathrm{W}$ are given by Seidelmann et al. (2005));

$$
r(a)=\left(\begin{array}{ccc}
\cos a & \sin a & 0 \\
-\sin a & \cos a & 0 \\
0 & 0 & 1
\end{array}\right), p(a)=\left(\begin{array}{ccc}
1 & 0 & 0 \\
0 & \cos a & \sin a \\
0 & -\sin a & \cos a
\end{array}\right) .
$$

The transformation in previous investigation (Eroshkin and Pashkevich, 2009) by means of the following matrix product:

$$
\left(\begin{array}{c}
\sigma_{\psi} \\
\sigma_{\theta} \\
\sigma_{\varphi}
\end{array}\right)=\left(\begin{array}{c}
\sigma_{z^{*}}^{E c l} \\
\sigma_{x^{*}}^{E q u} \\
-\sigma_{z^{*}}^{E q u}
\end{array}\right),\left(\begin{array}{c}
\sigma_{x^{*}}^{E q u} \\
\sigma_{y^{*}}^{E q u} \\
\sigma_{z^{*}}^{E q u}
\end{array}\right)=p\left(-\varepsilon_{*}\right)\left(\begin{array}{c}
\sigma_{x^{*}}^{E c l} \\
\sigma_{y^{*}}^{E c l} \\
\sigma_{z^{*}}^{E l}
\end{array}\right),\left(\begin{array}{l}
\sigma_{x^{*}}^{E c l} \\
\sigma_{y^{*}}^{E c l} \\
\sigma_{z^{*}}^{E l}
\end{array}\right)=r\left(\gamma_{0} \gamma_{*}\right) p\left(\varepsilon_{0}\right) r(\Delta)\left(\begin{array}{c}
\sigma_{\mathrm{X}} \\
\sigma_{\mathrm{Y}} \\
\sigma_{\mathrm{Z}}
\end{array}\right),
$$

where $\sigma_{\psi}$ is the geodetic motion of the equator of a body on the fixed ecliptic J2000; $\sigma_{\theta}$ is the geodetic variation of the obliquity of the equator of a body to the fixed ecliptic J2000; $\sigma_{\varphi}$ is the projection of the angular velocity vector of the geodetic rotation of a body to the axis of its angular velocity vector of the rotation; index $E c l$ correspond to the fixed ecliptic of epoch J2000, index Equ correspond to the equator of body. For each body the values of these projections were determined over the time span from AD1000 to AD3000 with one day spacing by using the DE404/LE404 ephemeris. The most essential terms of the geodetic rotation were found by means of the least squares method and spectral analysis methods. Here was finished previouse investigation.

The projections of angular velocity vector on the main axes of the inertias of Solar system body are defined by the Euler kinematical equations (Woolard, 1953):

$$
\left.\left.\begin{array}{l}
\omega_{1}=-\dot{\theta} \cos \varphi-\dot{\psi} \sin \theta \sin \varphi \\
\omega_{2}=\dot{\theta} \sin \varphi-\dot{\psi} \sin \theta \cos \varphi \\
\omega_{3}=\dot{\psi} \cos \theta+\dot{\varphi}
\end{array}\right\} \Rightarrow \begin{array}{r}
\dot{\psi} \sin \theta=-\omega_{1} \sin \varphi-\omega_{2} \cos \varphi \\
\dot{\theta}=-\omega_{1} \cos \varphi+\omega_{2} \sin \varphi \\
\dot{\varphi}=\omega_{3}-\dot{\psi} \cos \theta
\end{array}\right\} .
$$

Here $\psi, \theta, \varphi$ are Euler angels, the dot denotes a differentiation with respect to time.

From the difference between the relativistic (2) and Newtonian angular velocity vectors of Solar system body $\bar{\sigma}=\bar{\omega}_{R}-\bar{\omega}=\Delta \bar{\omega}$ is received the following expression:

$$
\left.\begin{array}{rl}
\dot{\psi}_{R} \sin \theta_{R}-\dot{\psi} \sin \theta & =-\omega_{R 1} \sin \varphi_{R}+\omega_{1} \sin \varphi-\omega_{R 2} \cos \varphi_{R}+\omega_{2} \cos \varphi \\
\Delta \dot{\theta} & =-\omega_{R 1} \cos \varphi_{R}+\omega_{1} \cos \varphi+\omega_{R 2} \sin \varphi_{R}-\omega_{2} \sin \varphi \\
\Delta \dot{\varphi} & =\Delta \omega_{3}-\dot{\psi}_{R} \cos \theta_{R}+\dot{\psi} \cos \theta
\end{array}\right\} .
$$

Here and further $\psi, \theta, \varphi$ are Euler angels in Newtonian case and $\psi_{R}, \theta_{R}, \varphi_{R}$ are Euler angels in relativistic case, $\Delta \dot{\psi}=\dot{\psi}_{R}-\dot{\psi} ; \Delta \dot{\theta}=\dot{\theta}_{R}-\dot{\theta} ; \Delta \dot{\varphi}=\dot{\varphi}_{R}-\dot{\varphi}$ are the differences between the 
relativistic and Newtonian components of the angular velocity vectors of Solar system body. In this study, it is considered that:

$$
\begin{aligned}
& \varphi_{R}=\varphi+\Delta \varphi \Rightarrow \sin (\varphi+\Delta \varphi)=\sin (\varphi) \cos (\Delta \varphi)+\sin (\Delta \varphi) \cos (\varphi) \approx \sin (\varphi) \\
& \cos (\varphi+\Delta \varphi)=\cos (\varphi) \cos (\Delta \varphi)-\sin (\Delta \varphi) \sin (\varphi) \approx \cos (\varphi) \\
& \theta_{R}=\theta+\Delta \theta \Rightarrow \sin (\theta+\Delta \theta)=\sin (\theta) \cos (\Delta \theta)+\sin (\Delta \theta) \cos (\theta) \approx \sin (\theta) \\
& \cos (\theta+\Delta \theta)=\cos (\theta) \cos (\Delta \theta)-\sin (\Delta \theta) \sin (\theta) \approx \cos (\theta)
\end{aligned}
$$

As a result from (8) and (9), following expressions are obtained:

$$
\left.\begin{array}{rl}
\Delta \dot{\psi} \sin \theta & =-\Delta \omega_{1} \sin \varphi-\Delta \omega_{2} \cos \varphi \\
\Delta \dot{\theta} & =-\Delta \omega_{1} \cos \varphi+\Delta \omega_{2} \sin \varphi \\
\Delta \dot{\varphi} & =\Delta \omega_{3}-\Delta \dot{\psi} \cos \varphi
\end{array}\right\} .
$$

Expressions for the perturbing terms of the physical librations of the Moon for the fixed ecliptic of epoch J2000 are defined by the following (Pashkevich and Eroshkin, 2011):

$$
\left.\begin{array}{l}
\tau=\varphi+\psi-180^{\circ}-L_{\mathbb{C}} \\
\rho=\theta-I \\
\sigma=\psi-\Omega
\end{array}\right\}
$$

where $\tau, \rho$ and $\sigma$ are the perturbing terms of the physical librations of the Moon in the longitude, in the inclination and in the node longitude, respectively; $\psi$ is the longitude of the descending node of epoch $\mathrm{J} 2000$ of the lunar equator; $I$ is a constant angle of the inclination of the lunar equator to the fixed ecliptic $\mathrm{J} 2000\left(I \sim 1^{\mathrm{O}} 32^{\prime}\right)$; $\theta$ is the inclination of the lunar equator to the fixed ecliptic J2000; $\varphi$ is the proper rotation angle of the Moon between the descending node of epoch $\mathrm{J} 2000$ and the principal axis of the minimum moment of inertia; $L_{\mathbb{}}$ is the mean longitude of the Moon and $\Omega$ is the mean longitude of the ascending node of its orbit.

As results of the elementary transformations from (10) and (11) the expressions of the velocities geodetic rotation are defined in the perturbing terms of the physical librations for the Moon (left 12) and in Euler angles for others Solar system bodies (right 12) as follows:

$$
\left.\left.\begin{array}{c}
\sin \theta \Delta \dot{\psi}=-\sigma_{1} \sin \varphi-\sigma_{2} \cos \varphi=\Delta(I \dot{\sigma}) \\
\Delta \dot{\theta}=-\sigma_{1} \cos \varphi+\sigma_{2} \sin \varphi=\Delta \dot{\rho} \\
\Delta \dot{\psi}+\Delta \dot{\varphi}=\sigma_{3}-\left(\sigma_{1} \sin \varphi+\sigma_{2} \cos \varphi\right) \tan \frac{\theta}{2}=\Delta \dot{\tau}
\end{array}\right\}, \begin{array}{l}
\Delta \dot{\psi}=-\frac{\sigma_{1} \sin \varphi+\sigma_{2} \cos \varphi}{\sin \theta} \\
\Delta \dot{\theta}=-\sigma_{1} \cos \varphi+\sigma_{2} \sin \varphi \\
\Delta \dot{\varphi}=\sigma_{3}-\Delta \dot{\psi} \cos \theta
\end{array}\right\} .
$$

Here the dot denotes a differentiation with respect to time.

B. The values of the velocities geodetic rotation are determined (in the perturbing terms of the physical librations for the Moon and in Euler angles for others Solar system bodies) over the time span from AD1000 to AD3000 with one day spacing by using the DE422/LE422 ephemeris. The behavior of these values of the velocities geodetic rotation for the major planets, Pluto, the Moon and the Sun are depicted in Figures $2-12$. 


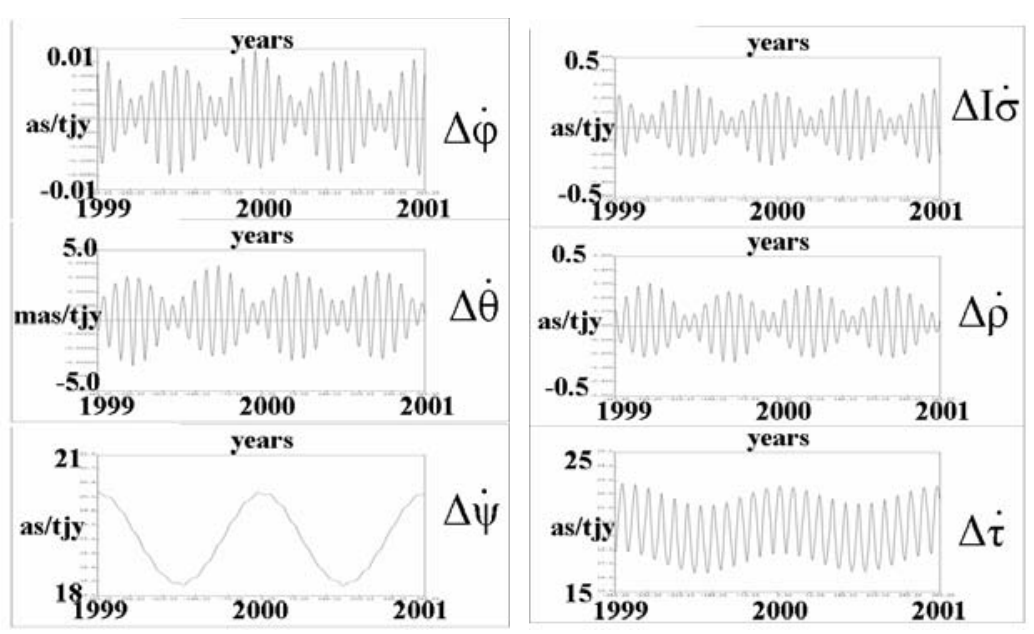

Fig. 2. The Earth (fragment) Fig. 3. The Moon (fragment)

Since the mass of the Sun is dominant in the solar system then the main part of the angular velocity vector of the geodetic rotation $\bar{\sigma}$ for each major planet and the Moon is a result of the heliocentric orbital motion of these bodies. The geodetic rotation of the Moon is determined not only by the Sun but also by the Earth. Figure 3 demonstrates it visually.

Some asymmetry of the components of the angular velocity vector of the geodetic rotation for Mercury (presented in Figure 4) and for Pluto (presented in Figure 5) is explained by the relatively large eccentricity of their orbits (e) as compared to the other planet orbits (Table 1). The sharp peaks of the curve correspond to Mercury's and Pluto's transits via perihelia. The values of the eccentricities for Mercury orbit and for Pluto orbit are close to each other. This fact explains a similar character of the behavior of the components $\Delta \dot{\psi}$ and $\Delta \dot{\varphi}$ for these planets. Since Mercury is the nearest planet to the Sun then it is clear that its geodetic rotation has to be the most significant in the solar system.

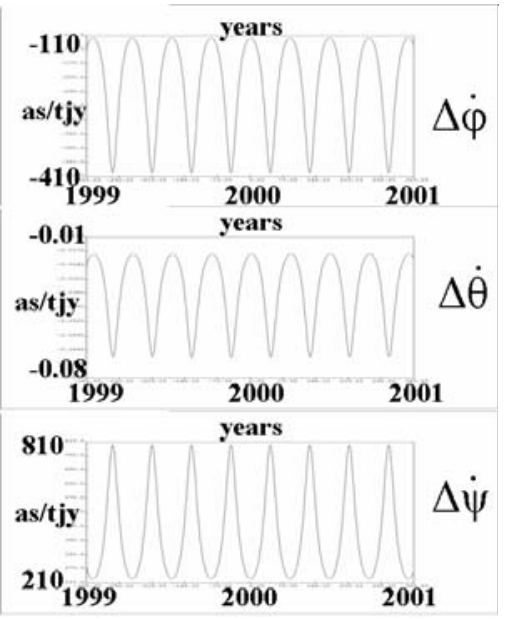

Fig. 4. Mercury (fragment)

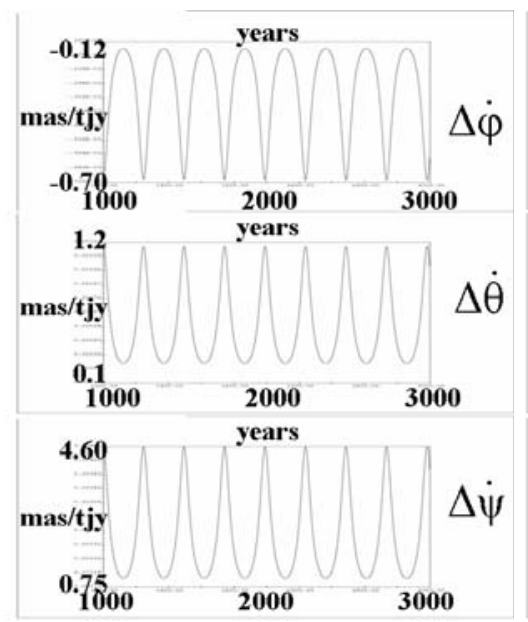

Fig. 5. Pluto

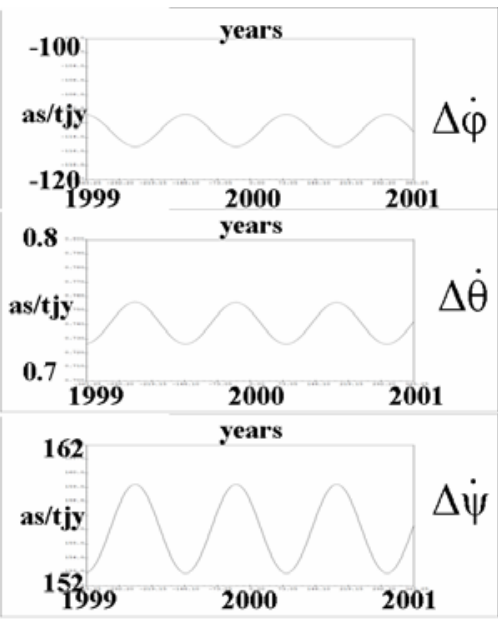

Fig. 6. Venus (fragment) 


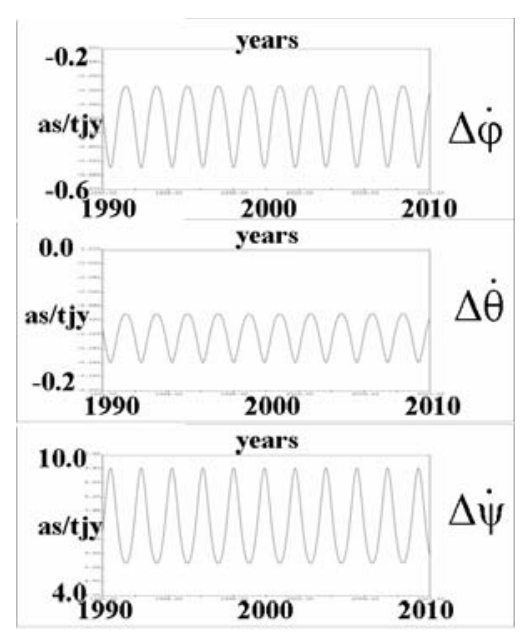

Fig. 7. Mars (fragment)

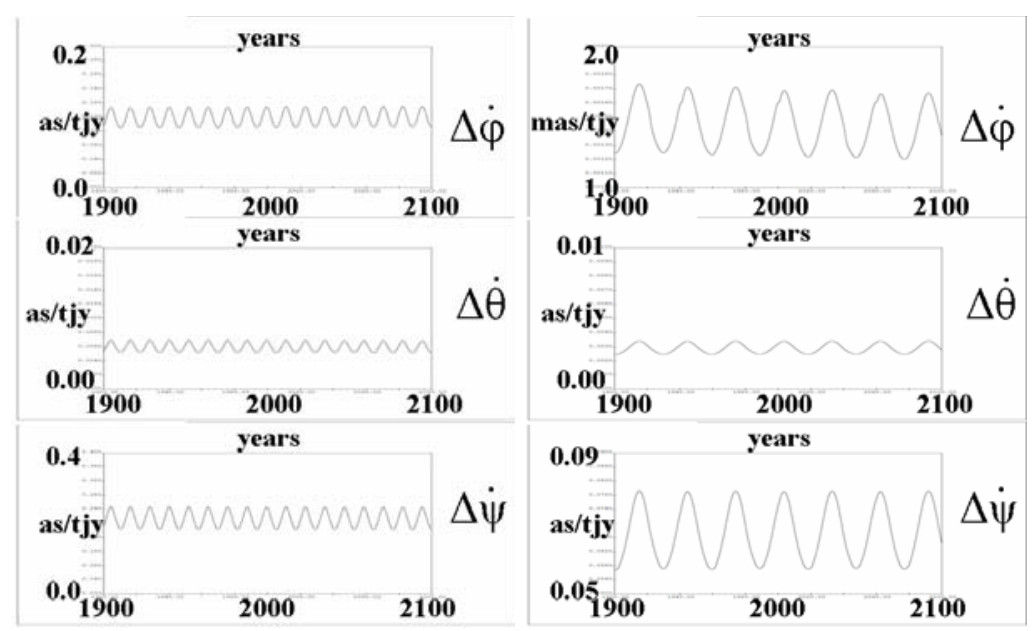

Fig. 8. Jupiter (fragment)

Fig. 9. Saturn (fragment)

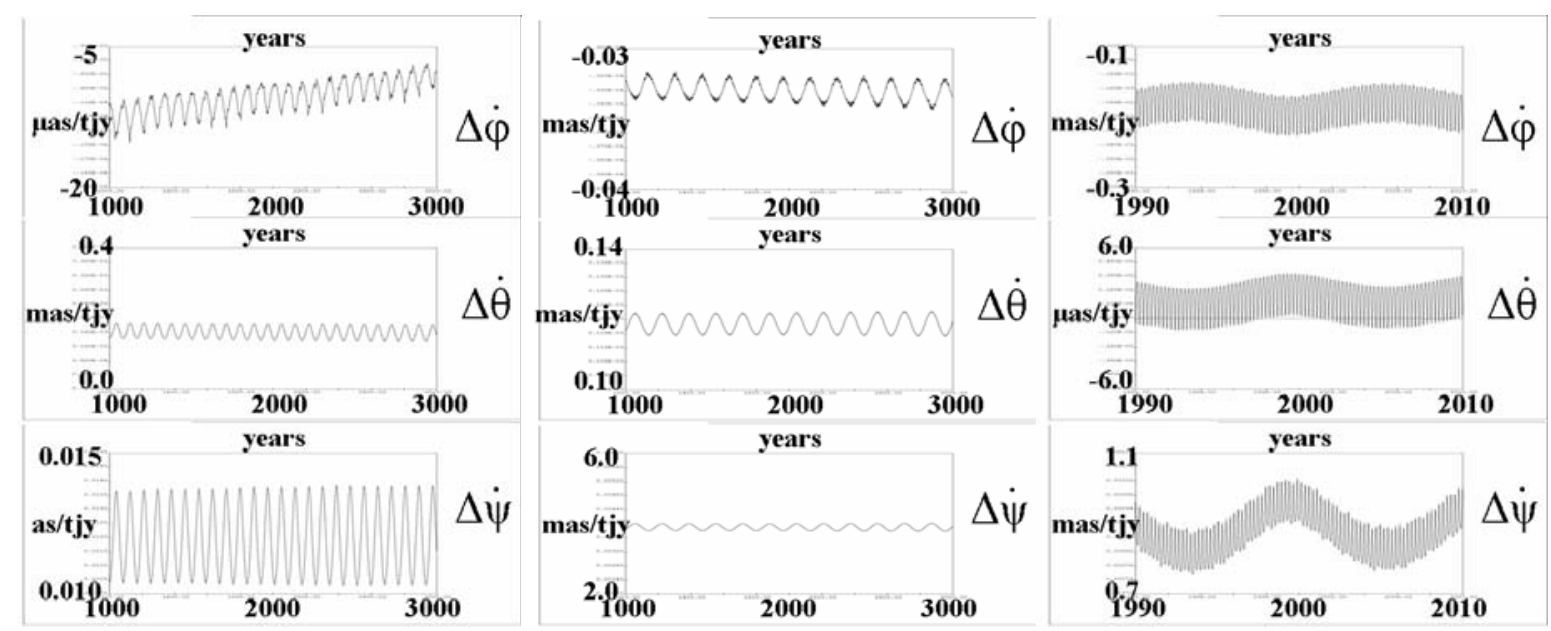

Fig. 10. Uranus

Fig. 12. The Sun (fragment)

The vector of the geodetic rotation of the Sun is determined by the orbital motion of the planets. Since the masses of the planets are essentially less than the mass of the Sun then the geodetic rotation of the Sun is very small. Its main part (presented in Figure 12) depends on the orbital motions of Jupiter, as the heaviest planet in the solar system, and Mercury, as the nearest planet to the Sun.

C. The most essential terms of the geodetic rotation are found by means of the least squares method and spectral analysis methods. The systematic and periodic terms of the body velocities geodetic rotation are presented by the following expressions:

$$
\Delta \dot{x}=\sum_{n=1}^{3} \Delta \dot{x}_{n} t^{n-1}+\sum_{i} \sum_{k=0}^{4}\left(\Delta \dot{x}_{C i k} \cos \left(v_{i 0}+v_{i 1} t\right)+\Delta \dot{x}_{S i k} \sin \left(v_{i 0}+v_{i 1} t\right)\right) t^{k}
$$

where $\Delta \dot{x}_{n}$ are the coefficients of the systematic terms; $\Delta \dot{x}_{C i k} \Delta \dot{x}_{S i k}$ are the coefficients of the periodic terms; $\dot{x}=\dot{\psi}, \dot{\theta}, \dot{\varphi}, \dot{\tau}, \dot{\rho}, \mathrm{I} \dot{\sigma} ; v_{i 0}, v_{i 1}$ are phases and frequencies of the body, which taken from (Brumberg and Bretagnon, 2000) and (Eroshkin and Pashkevich, 2007); $t$ is the time in the Julian days. After analytical integration (11) $\Delta x=\int \Delta \dot{x} \mathrm{dt}$ and application of "Cascade" method: 


$$
\begin{aligned}
& \Delta x_{S i m}=\frac{\Delta \dot{x}_{C i m}}{v_{i 1}}, \quad \Delta x_{C i m}=\frac{-\Delta \dot{x}_{S i m}}{v_{i 1}}, \\
& \Delta x_{S i m-1}=\frac{\Delta \dot{x}_{C i m-1}-m \Delta x_{C i m}}{v_{i 1}}, \Delta x_{C i m-1}=\frac{m \Delta x_{S i m}-\Delta \dot{x}_{S i m-1}}{v_{i 1}} \text {, } \\
& \Delta x_{S i 1}=\frac{\Delta \dot{x}_{C i 1}-2 \Delta x_{C i 2}}{v_{i 1}}, \quad \Delta x_{C i 1}=\frac{2 \Delta x_{S i 2}-\Delta \dot{x}_{S i 1}}{v_{i 1}}, \\
& \Delta x_{S i 0}=\frac{\Delta \dot{x}_{C i 0}-\Delta x_{C i 1}}{v_{i 1}}, \quad \Delta x_{C i 0}=\frac{\Delta x_{S i 1}-\Delta \dot{x}_{S i 0}}{v_{i 1}},
\end{aligned}
$$

(where $m=4$ ) are received the expressions of the systematic and periodic terms of the body geodetic rotation:

$$
\Delta x=\sum_{n=1}^{3} \frac{\Delta \dot{x}_{n}}{n} t^{n}+\sum_{i} \sum_{k=0}^{4}\left(\Delta x_{C i k} \cos A r g_{i}+\Delta x_{S i k} \sin \operatorname{Arg}_{i}\right) t^{k},
$$

where $\dot{x}=\dot{\psi}, \dot{\theta}, \dot{\varphi}, \dot{\tau}, \dot{\rho}, \mathrm{I} \dot{\sigma} ; x=\psi, \theta, \varphi, \tau, \rho, \mathrm{I} \sigma$.

\section{RESULTS}

As stated above the previous studies of the geodetic rotation of Solar system bodies were based on the JPL ephemeris DE404/LE404. The new calculations in this investigation are made on the basis of the improved JPL ephemeris DE422/LE422. The effect of the change of the values of geodetic rotation from using of the different ephemeris in Euler angles is smaller than $100 \mu$ as per thousand years for Mercury, as the body of Solar system with most values of the geodetic rotation, smaller than $20 \mu$ as per thousand years for Venus, smaller than $0.5 \mu$ as per thousand years for the Earth, Mars, smaller than 5 mas per thousand years for Jupiter, smaller than $0.05 \mu$ as per thousand years for Saturn, smaller than $0.005 \mu$ as per thousand years for Uranus, smaller than $0.01 \mu$ as per thousand years for Neptune, smaller than $0.02 \mu$ as per thousand years for and Pluto, and smaller than $0.0003 \mu$ as per thousand years for the Sun, as one body with least values of the geodetic rotation, and in the perturbing terms of the physical librations does not exceed $0.2 \mu$ as per thousand years for the Moon.

The values of the systematic (Table 1) and periodic (Table 2) terms of the body geodetic rotation were calculated a new above-mentioned method with using ephemeris DE422/LE422. 
Table 1. The systematic terms of geodetic rotation.

\begin{tabular}{|c|c|c|c|c|}
\hline & $\begin{array}{l}\text { Mercury } \\
\mathrm{e}=0.206\end{array}$ & $\begin{array}{c}\text { Venus } \\
\mathrm{e}=0.007\end{array}$ & $\begin{array}{c}\text { The Earth } \\
\text { (Brumberg and } \\
\text { Bretagnon, 2000) }\end{array}$ & $\begin{array}{l}\text { The Earth } \\
\mathrm{e}=0.017\end{array}$ \\
\hline & $\Delta \psi(\mu \mathrm{as})$ & $\psi($ (uas) & $\Delta \psi($ (uas) & $\Delta \psi($ uas $)$ \\
\hline $\mathrm{T}$ & 426451871.1763 & 156031996.8457 & 191988273.44 & 19198873.9203 \\
\hline $\mathrm{T}^{2}$ & 42516.8587 & 686532.2861 & -50386.32 & -50431.9734 \\
\hline \multirow[t]{2}{*}{$\mathrm{T}^{3}$} & -31016 & -786 & -754.09 & 656.9733 \\
\hline & $\Delta \theta(\mu \mathrm{as})$ & $\Delta \theta(\mu \mathrm{as})$ & $\Delta \theta(\mu \mathrm{as})$ & $\Delta \theta(\mu \mathrm{as})$ \\
\hline $\mathrm{T}$ & -36012.9217 & 740859.4714 & 9.55 & 12.7208 \\
\hline $\mathrm{T}^{2}$ & 09 & -6022 & 1954.11 & 1951 \\
\hline \multirow[t]{2}{*}{$\mathrm{T}^{3}$} & 185 & -628 & -4721.80 & 4125.3775 \\
\hline & $\Delta \varphi(\mu \mathrm{as})$ & $\Delta \varphi(\mu \mathrm{as})$ & $\Delta \varphi(\mu \mathrm{as})$ & $\Delta \varphi(\mu \mathrm{as})$ \\
\hline $\mathrm{T}$ & -214756714.5660 & -113010584.0490 & 2.99 & 8.7591 \\
\hline $\mathrm{T}^{2}$ & & -6870 & 54771.03 & 54775.0582 \\
\hline \multirow[t]{2}{*}{$\mathrm{T}^{3}$} & 2137 & 7879 & 802.06 & -1244.9150 \\
\hline & $\begin{array}{l}\text { The Moon } \\
\mathrm{e}=0.055\end{array}$ & $\begin{array}{c}\text { Mars } \\
\mathrm{e}=0.093\end{array}$ & $\begin{array}{c}\text { Jupiter } \\
\mathrm{E}=0.048\end{array}$ & $\begin{array}{c}\text { Saturn } \\
\mathrm{e}=0.056\end{array}$ \\
\hline & $\Delta \tau(\mu \mathrm{as})$ & $\Delta \psi(\mu \mathrm{as})$ & $\Delta \psi(\mu \mathrm{as})$ & $\Delta \psi(\mu \mathrm{as})$ \\
\hline $\mathrm{T}$ & 19494124.5437 & 7114256 & 213015.3078 & 67188 \\
\hline $\mathrm{T}^{2}$ & & -10 & -3541.1286 & 0002 \\
\hline \multirow[t]{2}{*}{$\mathrm{T}^{3}$} & -565.0947 & -9163.2412 & -15.0136 & -17.0358 \\
\hline & $\Delta \rho(\mu \mathrm{as})$ & $\Delta \theta(\mu \mathrm{as})$ & $\Delta \theta(\mu \mathrm{as})$ & $\Delta \theta(\mu \mathrm{as})$ \\
\hline $\mathrm{T}$ & 300.5067 & -119872.4123 & 5967.0475 & 2897.7159 \\
\hline $\mathrm{T}^{2}$ & 37 & 8 & -144.1639 & 27.8318 \\
\hline \multirow[t]{2}{*}{$\mathrm{T}^{3}$} & 3126.0421 & 419 & 5.7500 & -4.7791 \\
\hline & $\Delta(\mathrm{I} \sigma)(\mu \mathrm{as})$ & $\Delta \varphi(\mu \mathrm{as})$ & $\Delta \varphi(\mu \mathrm{as})$ & $\Delta \varphi(\mu \mathrm{as})$ \\
\hline $\mathrm{T}$ & -6544.4452 & -405155.9058 & 98655.1845 & 1444.1122 \\
\hline $\mathrm{T}^{2}$ & & 115 & 3561.4952 & -137.5508 \\
\hline \multirow[t]{2}{*}{$\mathrm{T}^{3}$} & -27 & & 189.2404 & -3.7812 \\
\hline & $\begin{array}{c}\text { Uranus } \\
\mathrm{e}=0.046\end{array}$ & $\begin{array}{l}\text { Neptune } \\
\mathrm{e}=0.009\end{array}$ & $\begin{array}{c}\text { Pluto } \\
E=0.249\end{array}$ & The Sun \\
\hline & $\Delta \psi(\mu \mathrm{as})$ & $\Delta \psi(\mu \mathrm{as})$ & $\Delta \psi(\mu \mathrm{as})$ & $\Delta \psi(\mu a s)$ \\
\hline $\mathrm{T}$ & 11924 & 3903.9461 & 2091.7329 & 870.0239 \\
\hline $\mathrm{T}^{2}$ & 21 & 188 & 28.5113 & -1.3770 \\
\hline $\mathrm{T}^{3}$ & 24.8305 & -0.9936 & 63.8836 & 0.2568 \\
\hline & $\Delta \theta(\mu \mathrm{as})$ & $\Delta \theta(\mu \mathrm{as})$ & $\Delta \theta(\mu \mathrm{as})$ & $\Delta \theta(\mu \mathrm{as})$ \\
\hline $\mathrm{T}$ & 160.6 & 118.7263 & 532.3802 & 1.8890 \\
\hline $\mathrm{T}^{2}$ & -1.4159 & -0.0724 & 6.9555 & -0.0809 \\
\hline $\mathrm{T}^{3}$ & 0.4365 & -0.0356 & 16.2397 & 0.0080 \\
\hline & $\Delta \varphi(\mu \mathrm{as})$ & $\Delta \varphi(\mu \mathrm{as})$ & $\Delta \varphi(\mu \mathrm{as})$ & $\Delta \varphi(\mu \mathrm{as})$ \\
\hline $\mathrm{T}$ & -10.3257 & -33.0498 & -314.2461 & -179.5716 \\
\hline $\mathrm{T}^{2}$ & 1.0611 & -0.1043 & -4.7359 & 1.3915 \\
\hline $\mathrm{T}^{3}$ & -0.0074 & 0.0312 & -9.6817 & -0.0433 \\
\hline
\end{tabular}


Table 2 (part one). The periodic terms of geodetic rotation.

\begin{tabular}{|c|c|c|}
\hline \multirow{3}{*}{ Mercury } & $\Delta \psi(\mu \mathrm{as})$ & $(2155.599-266.735 \mathrm{~T}+\ldots) \sin \lambda_{1}+(-9688.162-70.814 \mathrm{~T}+\ldots) \cos \lambda_{1}+\ldots$ \\
\hline & $\Delta \theta(\mu \mathrm{as})$ & $(-0.182+0.053 \mathrm{~T}+\ldots) \sin \lambda 1+(0.819-0.128 \mathrm{~T}+\ldots) \cos \lambda 1+\ldots$ \\
\hline & $\Delta \varphi(\mu \mathrm{as})$ & $\begin{array}{l}(-1085.536+134.503 \mathrm{~T}+\ldots) \sin \lambda_{1}+(4878.862 \\
+34.862 \mathrm{~T}+\ldots) \cos \lambda_{1}+\ldots\end{array}$ \\
\hline \multirow{3}{*}{ Venus } & $\Delta \psi(\mu \mathrm{as})$ & $(-205.908+12.505 \mathrm{~T}+\ldots) \sin \lambda_{2}+(-232.214+14.500 \mathrm{~T}+\ldots) \cos \lambda 2^{+} \ldots$ \\
\hline & $\Delta \theta$ ( $\mu$ as $)$ & $(-0.978+0.227 \mathrm{~T}+\ldots) \sin \lambda_{2}+(-1.103+0.258 \mathrm{~T}+\ldots) \cos \lambda_{2}+\ldots$ \\
\hline & $\Delta \varphi(\mu \mathrm{as})$ & $(149.135-8.556 \mathrm{~T}+\ldots) \sin \lambda 2^{+}(168.187-9.937 \mathrm{~T}+\ldots) \cos \lambda 2^{+} \ldots$ \\
\hline \multirow{3}{*}{$\begin{array}{l}\text { The Earth } \\
\text { (Brumberg } \\
\text { and } \\
\text { Bretagnon, } \\
2000 \text { ) }\end{array}$} & $\Delta \psi(\mu \mathrm{as})$ & $\begin{array}{l}(-34.28-7.36 \mathrm{~T}+\ldots) \sin \lambda_{3}+(-149.22+6.47 \mathrm{~T}+\ldots) \cos \lambda_{3}+ \\
(3.01+\ldots) \sin \left(\lambda_{3}+D-F\right)+(0.73 \mathrm{~T}+\ldots) \cos \left(\lambda_{3}+D-F\right)+\ldots\end{array}$ \\
\hline & $\Delta \theta$ ( $\mu$ as $)$ & $\begin{array}{l}(-0.03 \mathrm{~T}+\ldots) \cos \lambda 3^{+} \\
(0.32 \mathrm{~T}+\ldots) \sin \left(\lambda 3^{+} D-F\right)+(-1.30+\ldots) \cos \left(\lambda_{3}+D-F\right)+\ldots\end{array}$ \\
\hline & $\Delta \varphi(\mu$ as $)$ & $\begin{array}{l}(-0.20 \mathrm{~T}+\ldots) \sin \lambda_{3}+(-0.85 \mathrm{~T}+\ldots) \cos \lambda_{3}+ \\
(-3.28+\ldots) \sin (\lambda 3+D-F)+(-0.8 \mathrm{~T}+\ldots) \cos \left(\lambda_{3}+D-F\right)+\ldots\end{array}$ \\
\hline \multirow{3}{*}{ The Earth } & $\Delta \psi(\mu \mathrm{as})$ & $\begin{array}{l}(-34.284-7.360 \mathrm{~T}+\ldots) \sin \lambda_{3}+(-149.222+6.464 \mathrm{~T}+\ldots) \cos \lambda 3^{+} \\
(3.020-0.015 \mathrm{~T}+\ldots) \sin \left(\lambda_{3}+D-F\right)+ \\
(0.015-0.747 \mathrm{~T}+\ldots) \cos \left(\lambda_{3}+D-F\right)+\ldots\end{array}$ \\
\hline & $\Delta \theta(\mu \mathrm{as})$ & $\begin{array}{l}\left(3 \cdot 10^{-5}-0.007 \mathrm{~T}+\ldots\right) \sin \lambda_{3}+\left(-2 \cdot 10^{-5}-0.030 \mathrm{~T}+\ldots\right) \cos \lambda_{3}+ \\
\left(5 \cdot 10^{-4}-0.317 \mathrm{~T}+\ldots\right) \sin \left(\lambda_{3}+D-F\right)+ \\
\left(-1.301-7 \cdot 10^{-4} \mathrm{~T}+\ldots\right) \cos \left(\lambda_{3}+D-F\right)+\ldots\end{array}$ \\
\hline & $\Delta \varphi(\mu \mathrm{as})$ & $\begin{array}{l}\left(1 \cdot 10^{-4}-0.196 \mathrm{~T}+\ldots\right) \sin \lambda_{3}+\left(-1 \cdot 10^{-4}-0.851 \mathrm{~T}+\ldots\right) \cos \lambda_{3}+ \\
(-3.273-0.004 \mathrm{~T}+\ldots) \sin \left(\lambda_{3}+D-F\right)+ \\
(0.001+0.808 \mathrm{~T}+\ldots) \cos \left(\lambda_{3}+D-F\right)+\ldots\end{array}$ \\
\hline \multirow{3}{*}{ The Moon } & $\Delta \tau$ ( $\mu$ as) & $\begin{array}{l}(-34.279-7.559 \mathrm{~T}+\ldots) \sin \lambda 3+(-149.201+5.683 \mathrm{~T}+\ldots) \cos \lambda 3+ \\
(30.212-0.001 \mathrm{~T}+\ldots) \sin D+(0.001+0.001 \mathrm{~T}+\ldots) \cos D+\ldots\end{array}$ \\
\hline & $\Delta \rho$ ( $\mu$ as) & $\begin{array}{l}\left(-9 \cdot 10^{-4}-0.008 \mathrm{~T}+\ldots\right) \sin \lambda 3+\left(-3 \cdot 10^{-4}-0.025 \mathrm{~T}+\ldots\right) \cos \lambda 3+ \\
(-0.004+0.010 \mathrm{~T}+\ldots) \sin D+(0.005+0.007 \mathrm{~T}+\ldots) \cos D+\ldots\end{array}$ \\
\hline & $\Delta(\mathrm{I} \sigma)(\mu \mathrm{as})$ & $\begin{array}{l}(0.013-0.111 \mathrm{~T}+\ldots) \sin \lambda 3+(0.052-0.496 \mathrm{~T}+\ldots) \cos \lambda 3+ \\
(-0.016+0.093 \mathrm{~T}+\ldots) \sin D+(-0.006+0.004 \mathrm{~T}+\ldots) \cos D+\ldots\end{array}$ \\
\hline \multirow{3}{*}{ Mars } & $\Delta \psi(\mu \mathrm{as})$ & $(543.435+22.457 \mathrm{~T}+\ldots) \sin \lambda 4+(241.415-40.426 \mathrm{~T}+\ldots) \cos \lambda 4+\ldots$ \\
\hline & $\Delta \theta(\mu \mathrm{as})$ & $(-9.157-0.241 \mathrm{~T}+\ldots) \sin \lambda_{4}+(-4.068+0.742 \mathrm{~T}+\ldots) \cos \lambda 4+\ldots$ \\
\hline & $\Delta \varphi(\mu \mathrm{as})$ & $(-30.949+0.392 \mathrm{~T}+\ldots) \sin \lambda 4+(-13.748+3.044 \mathrm{~T}+\ldots) \cos \lambda 4+\ldots$ \\
\hline \multirow{3}{*}{ Jupiter } & $\Delta \psi(\mu \mathrm{as})$ & $(56.618-0.526 \mathrm{~T}+\ldots) \sin \lambda 5+(-14.560-2.255 \mathrm{~T}+\ldots) \cos \lambda 5+\ldots$ \\
\hline & $\Delta \theta$ ( $\mu$ as $)$ & $(1.587-0.039 \mathrm{~T}+\ldots) \sin \lambda 5+(-0.408-0.057 \mathrm{~T}+\ldots) \cos \lambda 5+\ldots$ \\
\hline & $\Delta \varphi(\mu \mathrm{as})$ & $(26.227+2.506 \mathrm{~T}+\ldots) \sin \lambda 5+(-6.739-1.722 \mathrm{~T}+\ldots) \cos \lambda 5+\ldots$ \\
\hline \multirow{3}{*}{ Saturn } & $\Delta \psi(\mu \mathrm{as})$ & $(-2.688-5.015 \mathrm{~T}+\ldots) \sin \lambda_{6}+(-52.010+3.313 \mathrm{~T}+\ldots) \cos \lambda_{6}+\ldots$ \\
\hline & $\Delta \theta(\mu \mathrm{as})$ & $(-0.115-0.219 \mathrm{~T}+\ldots) \sin \lambda 6+(-2.242+0.102 \mathrm{~T}+\ldots) \cos \lambda 6+\ldots$ \\
\hline & $\Delta \varphi(\mu \mathrm{as})$ & $(-0.061-0.097 \mathrm{~T}+\ldots) \sin \lambda_{6}+(-1.119+0.291 \mathrm{~T}+\ldots) \cos \lambda_{6}+\ldots$ \\
\hline \multirow{3}{*}{ Uranus } & $\Delta \psi(\mu \mathrm{as})$ & $(-22.392-1.432 \mathrm{~T}+\ldots) \sin \lambda 7+(-3.418+0.773 \mathrm{~T}+\ldots) \cos \lambda 7+\ldots$ \\
\hline & $\Delta \theta$ ( $\mu$ as $)$ & $(-0.302-0.012 \mathrm{~T}+\ldots) \sin \lambda 7+(-0.046+0.012 \mathrm{~T}+\ldots) \cos \lambda 7+\ldots$ \\
\hline & $\Delta \varphi(\mu \mathrm{as})$ & $(0.019-0.002 \mathrm{~T}+\ldots) \sin \lambda 7+(0.003-0.002 \mathrm{~T}+\ldots) \cos \lambda 7+\ldots$ \\
\hline
\end{tabular}


Table 2 (part two). The periodic terms of geodetic rotation.

\begin{tabular}{|c|c|c|}
\hline \multirow{3}{*}{ Neptune } & $\Delta \psi(\mu \mathrm{as})$ & $(1.879+0.301 \mathrm{~T}+\ldots) \sin \lambda 8+(-1.829-0.066 \mathrm{~T}+\ldots) \cos \lambda 8+\ldots$ \\
\hline & $\Delta \theta(\mu \mathrm{as})$ & $(0.057+0.010 \mathrm{~T}+\ldots) \sin \lambda 8+(-0.056-0.002 \mathrm{~T}+\ldots) \cos \lambda 8+\ldots$ \\
\hline & $\Delta \varphi(\mu \mathrm{as})$ & $(-0.017-0.002 \mathrm{~T}+\ldots) \cos \lambda 8+(0.015+0.001 \mathrm{~T}+\ldots) \cos \lambda 8+\ldots$ \\
\hline \multirow{3}{*}{ Pluto } & $\Delta \psi(\mu \mathrm{as})$ & $(62.592-1.868 \mathrm{~T}+\ldots) \sin \lambda 9+(0.307+15.195 \mathrm{~T}+\ldots) \cos \lambda 9+\ldots$ \\
\hline & $\Delta \theta$ ( $\mu$ as $)$ & $(15.930-0.489 \mathrm{~T}+\ldots) \sin \lambda 9+(0.080+3.864 \mathrm{~T}+\ldots) \cos \lambda 9+\ldots$ \\
\hline & $\Delta \varphi(\mu \mathrm{as})$ & $(-9.404+0.256 \mathrm{~T}+\ldots) \sin \lambda 9+(-0.047-2.286 \mathrm{~T}+\ldots) \cos \lambda 9+\ldots$ \\
\hline \multirow{3}{*}{ The Sun } & $\Delta \psi(\mu \mathrm{as})$ & $\begin{array}{l}(0.123+0.003 \mathrm{~T}+\ldots) \sin \lambda_{5}+(-0.032-0.006 \mathrm{~T}+\ldots) \cos \lambda_{5}+ \\
\left(4 \cdot 10^{-4}-1 \cdot 10^{-4} \mathrm{~T}+\ldots\right) \sin \lambda_{1}+\left(-0.002+4 \cdot 10^{-6} \mathrm{~T}+\ldots\right) \cos \lambda_{1}+\ldots\end{array}$ \\
\hline & $\Delta \theta(\mu \mathrm{as})$ & $\begin{array}{l}\left(0.001+1 \cdot 10^{-4} \mathrm{~T}+\ldots\right) \sin \lambda_{5}+\left(-3 \cdot 10^{-4}-1 \cdot 10^{-4} \mathrm{~T}+\ldots\right) \cos \lambda_{5}+ \\
\left(-1 \cdot 10^{-5}+1 \cdot 10^{-6} \mathrm{~T}+\ldots\right) \sin \lambda_{1}+\left(1 \cdot 10^{-4}+2 \cdot 10^{-6} \mathrm{~T}+\ldots\right) \cos \lambda_{1}+\ldots\end{array}$ \\
\hline & $\Delta \varphi(\mu \mathrm{as})$ & $\begin{array}{l}\left(-0.017+1 \cdot 10^{-4} \mathrm{~T}+\ldots\right) \sin \lambda_{5}+(0.004+0.001 \mathrm{~T}+\ldots) \cos \lambda_{5}+ \\
\left(-2 \cdot 10^{-4}+3 \cdot 10^{-5} \mathrm{~T}+\ldots\right) \sin \lambda_{1}+\left(0.001-1 \cdot 10^{-5} \mathrm{~T}+\ldots\right) \cos \lambda_{1}+\ldots\end{array}$ \\
\hline
\end{tabular}

In Table 1 and Table 2: $D=\lambda_{10}-\lambda_{3}+180^{\circ}, \lambda_{j}(j=1, \ldots, 9)$ are mean longitudes of the planets and Pluto; $\lambda_{10}$ is mean geocentric longitude of the Moon; e is eccentricity of Solar system bodies orbits; $\mathrm{T}$ is means the Dynamical Barycentric Time (TDB) measured in thousand Julian years (tjy) (of 365250 days) from J2000. The mean longitudes of the planets and the Moon were taken from (Brumberg and Bretagnon, 2000). The mean longitude of Pluto was taken from previous investigation (Eroshkin and Pashkevich, 2007).

It is easy to see that the values of the secular parts of the geodetic rotation of each body depend on its distance from the Sun (Table 1). For any body of the Solar system the periodic terms of the geodetic rotation depend not only on its distance from the Sun (Table 2), but also on the values of the eccentricities of their orbits (Table 1). So, since the value of the eccentricity for Mars is larger than that for the Earth then the amplitudes of the main periodic terms in the components of the geodetic rotation of Mars (Figure 7) are larger those that for the Earth (Figure 2).

The Table 1 and Table 2 show that that the systematic and periodic terms of the geodetic Earth's rotation this study coincides well with similar terms in Brumberg and Bretagnon (2000). The remarkable difference in the sign of the cubic terms in Table 1 is explained by the different theories of the orbital motions. So, the semi-analytical solution of Brumberg and Bretagnon (2000) is essentially based upon the semi-analytical theory of the rigid Earth rotation SMART97 by Bretagnon et al., (1998) and the semi-analytical theory of the orbital motion of the major planets VSOP87 by Bretagnon and Francou (1988), while the results of the present paper are based upon DE422/LE422 numerical ephemeris.

This fact demonstrates the effectiveness and reliability of the proposed new method for calculating the values of the quantities of geodetic rotation for any solar system body.

\section{CONCLUSIONS}

New method for calculation the values of the geodetic rotation any bodies of the Solar system was received. This method has a good confirmation of the results for the Earth case.

For the first time new high-precision values of the geodetic rotation of the major planets, Pluto and the Sun were obtained for Euler angles. 
For the first time new high-precision values of the geodetic rotation of the Moon ephemeris were obtained for the perturbing terms of the physical librations. These analytical values of the geodetic rotation of the Moon will be use for the numerical investigations of the Moon rotation in the relativistic approximation.

All the above analytical values of the geodetic rotation of the Solar system bodies are dynamically adjusted to the DE422/LE422 ephemeris.

This study confirms the all findings of our previous investigations (Eroshkin and Pashkevich, 2007) and (Eroshkin and Pashkevich, 2009):

The secular terms of geodetic rotation of each planet of the Solar system depend on their distance from the Sun, the mass of which is dominant in the solar system.

For any planet the periodic part of the geodetic rotation depends on its distance from the Sun and from the values of the eccentricities of their orbits.

The main periodic part of the geodetic rotation of the Moon is determined not only by the Sun but also by the Earth, which is the nearest planet to the Moon.

The geodetic rotation of the Sun depends not only on the orbital motion of Jupiter, as the heaviest planet in the solar system, but also on the orbital motion of Mercury which has the most rapid orbital motion.

For the Sun, giant planets and Pluto the geodetic rotation is insignificant.

For the terrestrial planets and the Moon the geodetic rotation is significant and has to be taken into account for the construction of the high-precision theories of the rotational motion of these bodies.

Geodetic rotation has to be taken into account if the influence of the dynamical figure of a body on its orbital-rotational motion is studied in the post-Newtonian approximation.

The lunar laser ranging data processing has to use the relativistic theory of the rotation of the Moon, as well as that of the Earth.

Acknowledgements. The investigation was carried out at the Central (Pulkovo) Astronomical Observatory of the Russian Academy of Science and the Space Research Centre of the Polish Academy of Science, under a financial support of the Cooperation between the Polish and Russian Academies of Sciences, Theme No 34 and Aleksander Brzeziński personal grant the Polish national science foundation NCN under grant No. DEC-2012/05/B/ST10/02132.

\section{REFERENCES}

Woolard E.W. (1953) Theory of the rotation of the Earth around its center of mass, in Astronomical Papers for the American Ephemeris and Nautical Almanac XV, Part 1, U.S. Government Printing Office, Washington.

Bretagnon P.and Francou G. (1988) Planetary theories in rectangular and spherical variables, Astronomy and Astrophysics, 202, pp. 309-315.

Standish E.M., Newhall X X, Williams J.G. and Folkner W.M. (1995) JPL Planetary and Lunar Ephemerides, DE403/LE403, JPL IOM 314.10-127.

Bretagnon P., Francou G., Rocher P., Simon J.L. (1998) SMART97: A new solution for the rotation of the rigid Earth, Astron. Astrophys. , 329, 1, pp. 329-338.

Brumberg V.A., Bretagnon P. (2000) Kinematical Relativistic Corrections for Earth's Rotation Parameters, in Proc. of IAU Colloquium 180, eds. K. Johnston, D. McCarthy, B. Luzum and G. Kaplan, U.S. Naval Observatory, pp. 293-302. 
Seidelmann P.K., Archinal B.A., A'Hearn M.F., Cruikshank D.P., Hilton J.L., Keller H.U., Oberst J., Simon J.L., Stooke P., Tholen D.J., and Thomas P.C. (2005) Report of the IAU/IAG Working Group on Cartographic Coordinates and Rotational Elements: 2003, Celestial Mechanics and Dynamical Astronomy, 91, pp. 203-215.

Eroshkin G.I., Pashkevich V.V. (2007) Geodetic rotation of the Solar system bodies, Artificial Satellites, Vol. 42, No. 1, pp. 59-70.

Eroshkin G.I., Pashkevich V.V. (2009) On the geodetic rotation of the major planets, the Moon and the Sun, Artificial Satellites, Vol. 44, No. 2, pp 43-52.

Folkner W.F. (2011) JPL Planetary and Lunar Ephemerides: Export Information http://iaucomm4. jpl.nasa.gov/README.html.

Pashkevich V.V., Eroshkin G.I. (2011) Construction of the new high-precision Moon rotation series at a long time intervals, Artificial Satellites, Vol. 46. No. 2. (DOI: 10.2478/v10018-011-0013-3), pp. 63-73.

Received: 2015-11-27,

Reviewed: 2016-01-15, by V. Zharov, and 2016-04-04, by N. Petrova,

Accepted: 2016-05-04. 